\title{
Persistent mild hip deformation in preterm infants
}

\author{
P M Davis, R Robinson, L Harris, P H T Cartlidge
}

Department of Child Health, University of Wales College of Medicine, Cardiff P M Davis

R Robinson

L Harris

P H T Cartlidge

Correspondence to: Dr P M Davis, Department of Community Child Health, of Community Child Health, Community Health Unit, Lansdowne Hospital, Canton, Cardiff CF1 8UL.

Accepted 28 June 1993

\begin{abstract}
Preterm infants often develop a postural deformation of the legs with wide hip abduction and external rotation giving a 'frog leg' posture. It has been shown that the deformation persists at the age of $3-4 \cdot 5$ years and the use of postural techniques aimed at reducing the deformation is recommended.
\end{abstract}

(Arch Dis Child 1993; 69: 597-598)

The global hypotonia and immobility of preterm infants enables gravity to have a marked effect on posture, resulting in the deformation of malleable tissues. The moulding predominantly affects the head which becomes flattened; the shoulders with retraction and abduction; and lower limbs with wide hip abduction and external rotation giving a 'frog leg' posture. ${ }^{1-3}$ Deformation of the hip is thought to disadvantage later weight bearing, particularly in neurologically impaired children, due to the associated shortening of the adductor magnus muscle. ${ }^{2}$

Postnatal moulding can be reduced. Head flattening, due to nursing preterm babies on a firm mattress, is lessened by nursing these infants on a soft air mattress or by using a water pillow during the first weeks of life. ${ }^{14}$ However, when the child is able to move the head more easily, and with growth and remodelling, the difference lessens so that by 3-4 years of age head shape is normal regardless of the type of mattress used in the newborn period. ${ }^{5}$ Downs et al showed that hip deformation can also be reduced in very preterm infants by postural intervention during the first weeks of life. ${ }^{2}$ However, infants in her study were assessed at 40 weeks' postmenstrual age and it is not known whether the benefit is maintained. Furthermore, it is not known whether with increased mobility and remodelling the deformity resolves regardless of intervention. The aim of our study was to investigate whether the postnatal hip deformation seen in preterm infants persisted into childhood.

weeks' gestation or more and attending local nurseries. These infants were also nursed predominantly prone. None of the children had a neurological deficit, congenital anomaly, or breech delivery. Hip deformation was assessed in two ways. Footprint angles (the degree of out-toeing or in-toeing) was measured by the child walking briskly along a strip of paper with talcum powder on the soles of the feet, footprints were traced, and a line was drawn along the medial border of each foot. The angle of intersection was designated positive for in-toeing and negative for out-toeing. Hip rotation was measured using standard clinical method. The child lay prone with the knee flexed to a right angle in the neutral position, internal and external rotation of each limb was measured separately using a goniometer and summated to give the total arc of rotation. The mean value for the two limbs was then calculated.

Statistical analysis was by the Kruskal-Wallis test and $\mathrm{F}$ test for equality of variances. The study was approved by the district ethics committee.

\section{Results}

Out-toeing was significantly more common in the preterm group, occurring in $62 \%$ of these children compared with only $35 \%$ in the term group (fig 1). The mean (SD) footprint angle in the preterm group was $-4.5^{\circ}(11 \cdot 8)$ (outtoeing) compared with $+8 \cdot 2^{\circ}(14 \cdot 3)$ (in-toeing) in the term group $(p<0.02)$. The large variance in each group indicates that no degree of in-toeing or out-toeing can be considered abnormal.

The total arc of rotation at the hip was significantly greater in the preterm group, with a mean (SD) of $119 \cdot 2^{\circ}(19 \cdot 6)$ compared with $99 \cdot 2^{\circ}(9 \cdot 6)$ in the term group $(p<0.003$; fig 2$)$. The difference resulted from an increase in both internal and external rotation. Internal rotation was greater in the preterm group $-60 \cdot 4^{\circ}(15 \cdot 6)$ compared with $50.9^{\circ}(9.5)$ in the term group, but this did not reach statistical significance $(p=0.07)$. External rotation

\section{Subjects and methods}

Thirty three children aged 3-4.5 years were included in the study. The preterm group consisted of 13 children (six girls, seven boys) with an original gestational age of 26-32 weeks (median 28 weeks) and birth weight 850$2090 \mathrm{~g}$ (median $1040 \mathrm{~g}$ ). They were born at the University Hospital of Wales or Llandough Hospital where unit policy in both was to nurse preterm babies prone during the newborn period. The control group comprised 20 children (12 girls, eight boys) born at 38

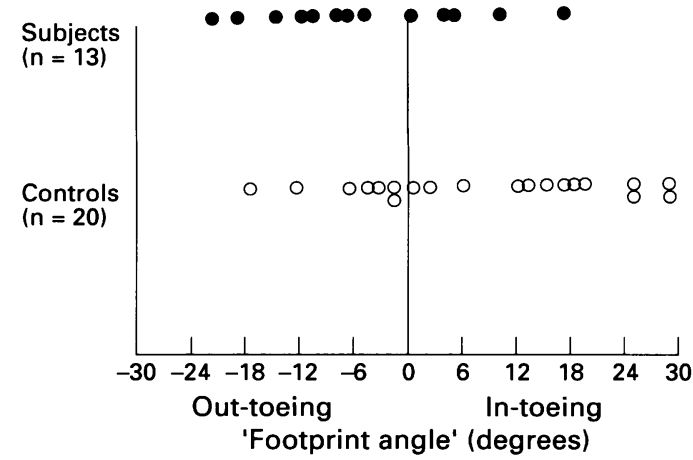

Figure 1 Out-toeing in preterm and control groups. 


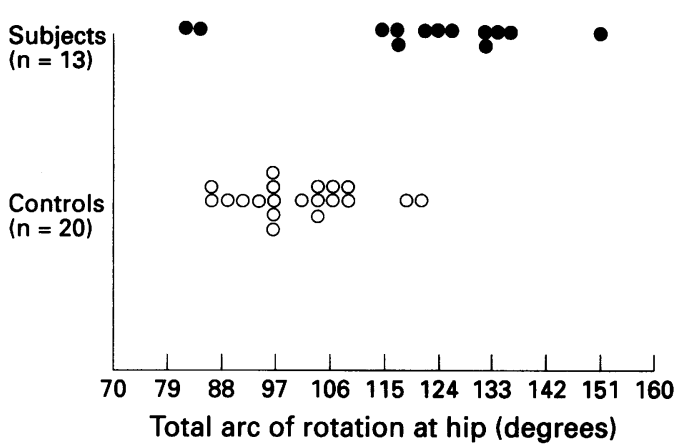

Figure 2 Total arc of rotation of the hip in preterm and control groups.

was also greater in the preterm group with a mean (SD) of $58 \cdot 8^{\circ}(22 \cdot 8) v 48 \cdot 4^{\circ}(7 \cdot 8)$, but again this was not significant $(\mathrm{p}=0.062$; fig 3 ). The two populations differed markedly with respect to the spread of values for hip mobility. The ratio of the variances between control and subject groups for total rotation was $4 \cdot 14$ $(p<0.01)$ and for external rotation 8.54 $(p<0.001)$. This suggests that a variety of deforming forces act at different times on the newborn infant, resulting in excess internal rotation in some and external rotation in others.

\section{Discussion}

We have shown that the deformation of the lower limb frequently seen in preterm babies during early infancy has not resolved by the age of 3-4.5 years. The out-toeing may in part be accounted for by increased external rotation of the hip, although this did not reach significance. In addition, deforming forces are probably producing torsion of the rest of the leg, and also the ankle which has been noted to adopt a position of eversion in very preterm infants. ${ }^{3}$ The range of hip mobility in children born prematurely is also increased. This is probably due to increased joint laxity caused by deforming forces acting on the hip at a time when immobility and hypotonia prevent the body from resisting.

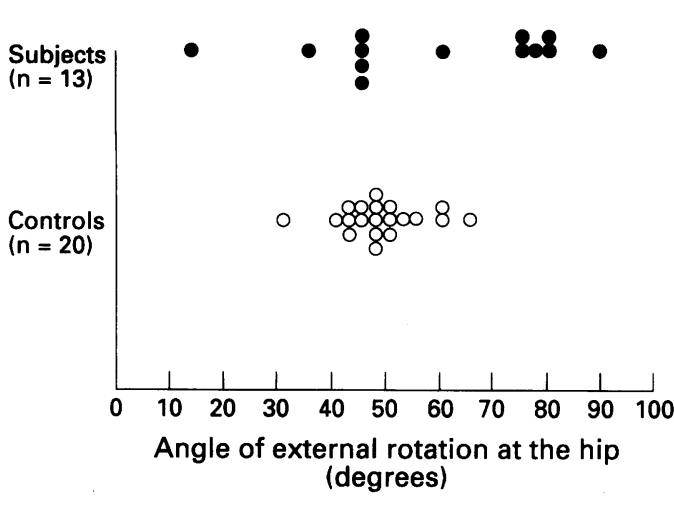

Figure 3 External rotation in preterm and control groups.

The deformation frequently worries parents who prefer their children to look like children born at term. ${ }^{6}$ Moreover, the abnormal posture may further impair development in children with neurological abnormalities. Whether the deformation impairs function in neurologically normal children is not known, although our impression is that walking is delayed due to difficulties in achieving good balance. We consider that frequent changes in position and postural techniques ${ }^{2}$ aimed at reducing the deformation should be used, but further studies are needed to establish the long term benefit of intervention.

We thank Mrs V Williams and Miss J Thomas for their help and advice and the parents, children, and staff of Treetops Community Playgroup in Lisvane and the creche at the University Hospital of Wales. We are indebted to Dr Newcombe for his statistical advice.

1 Cartlidge PHT, Rutter N. Reduction of head flattening in preterm infants. Arch Dis Child 1988; 63: 755-7.

2 Downs JA, Edwards DA, McCormick DC, Roth SC, Stewart AL. The effect of postural intervention on the development of hip posture in preterm babies $<33$ weeks gestation. Proceedings of the Neonatal Society, March 1990.

3 Updike C, Schmidt RE, Macke C, Cahoon J, Miller M. Positional support for premature infants. Am $\mathcal{f}$ Occup Ther 1986; 40: 712-5.

4 Marsden DJ. Reduction of head flattening in preterm infants. Dev Med Child Neurol 1980; 22: 507-9.

5 Rutter N, Hinchcliffe W, Cartlidge PHT. Do preterm infants always have flattened heads? Arch Dis Child 1993; 68: 606-7.

6 Budreau GK. Postnatal cranial moulding and infant attractiveness: implications for nursing. Neonatal Network 1987;
5: 13-9. 